\title{
ARTHROGRAPHY OF THE SHOULDER AFTER OPERATIVE REPAIR OF THE TORN ROTATOR CUFF
}

\author{
P. T. CALVERT, N. P. PACKER, D. J. STOKER, J. I. L. BAYLEY, L. KESSEL
}

From the Royal National Orthopaedic Hospital and the Institute of Orthopaedics, London

\begin{abstract}
Double-contrast shoulder arthrograms were performed in 20 patients at an average of $\mathbf{3 0}$ months after operative repair of a torn rotator cuff. In 18 out of 20 shoulders the contrast medium leaked into the subacromial bursa indicating a defect in the rotator cuff. Despite this, 17 patients had complete relief of pain and $15 \mathrm{had}$ a full range of shoulder elevation. The results suggest that a completely watertight closure is not essential for a good functional result, and that arthrography may not be helpful in the investigation of failure of repair.
\end{abstract}

The value of operative repair of the torn rotator cuff of the shoulder remains controversial. When surgical treatment is indicated a variety of materials and methods have been used in order to effect a complete repair. In some instances emphasis has been placed on making the repair "watertight" (Neviaser 1980). Little attention has been paid to whether this is essential for a good clinical result, or whether the repair remains radiologically intact.

In an attempt to answer these questions, shoulder arthrograms were performed on a series of patients who had had operative repair of a torn rotator cuff. The findings were then correlated with the clinical result.

\section{MATERIAL AND METHODS}

Twenty patients who had undergone operative repair of a torn rotator cuff of the shoulder agreed, when seen for clinical review, to have a double-contrast shoulder arthrogram. There were 16 men and four women with an average age of 58 years (range 41 to 75 years). The dominant side was affected in 12 patients. The main indication for operation had been persistent pain. This was present in 17 patients, seven of whom also had loss of active elevation. Two patients had loss of active elevation

P. T. Calvert, MA, FRCS, Consultant Orthopaedic Surgeon Hinchingbrooke Hospital. Hinchingbrooke Park, Huntingdon, Cambridgeshire PE18 8NT, England.

N. P. Packer, FRCS, Consultant Orthopaedic Surgeon

Queen Elizabeth Hospital, Gayton Road, King's Lynn, Norfolk PE30 4ET, England.

D. J. Stoker, FRCP, FRCR, Consultant Radiologist

J. I. L. Bayley, FRCS, Consultant Orthopaedic Surgeon, Shoulder Unit

L. Kessel, MBE, MC, FRCS, Emeritus Professor of Orthopaedics, Private Consulting Rooms

Royal National Orthopaedic Hospital, 45/51 Bolsover Street, London WIP 8AQ, England.

Requests for reprints should be sent to Mr P. T. Calvert.

(C) 1986 British Editorial Society of Bone and Joint Surgery $0301-620 \mathrm{X} / 86 / 1052 \$ 2.00$ without pain. One patient (Case 5) could achieve active elevation, but with an altered rhythm. He could not keep his arm above his shoulder for more than a few minutes; since he was employed as a plasterer this was a significant disability.

The details of all 20 patients are given in Table I. Table II summarises the operative findings and procedures.

All the arthrograms were performed by one consultant radiologist (DJS), who was not aware of the clinical assessment made by one of the authors (PTC or NPP). Arthrography was performed by a standard doublecontrast technique. Through an anterior approach a 22 -gauge spinal needle was used to inject 4 to $5 \mathrm{ml}$ of $60 \%$ meglumine iothalamate (Conray 280 ) into the joint, followed by $20 \mathrm{ml}$ of room air. The contrast media were injected under fluoroscopic control; where a defect in the rotator cuff was present, its size was assessed by the mode of passage of the medium through the defect. Immediate filling of the subacromial bursa indicated a large defect ( $2 \mathrm{~cm}$ or more). A leak characterised by the passage of a thin column of medium constituted a small defect ( $1 \mathrm{~cm}$ or less). Defects between these two extremes were classified as medium-sized.

At review the patients were asked about pain-relief and functional ability. The range of movement of each shoulder was recorded. The average length of follow-up between operation and review was 30 months (range 6 to 78 months). All arthrograms were performed within a few weeks of the clinical review.

\section{RESULTS}

In only two of the 20 shoulder arthrograms was the rotator cuff radiographically intact, as shown by the absence of any leak from the shoulder into the subacromial bursa. In the remaining 18 shoulders, contrast medium passed into the bursa indicating a defect in the rotator cuff. The estimated size of the defect was small in eight, 
P. T. CALVERT, N. P. PACKER, D. J. STOKER, J. I. L. BAYLEY, L. KESSEL

造点

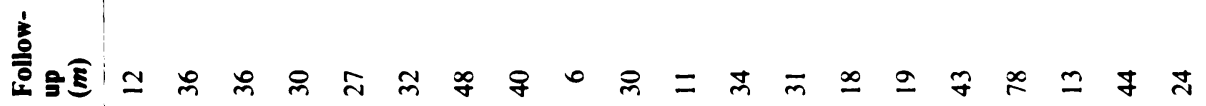

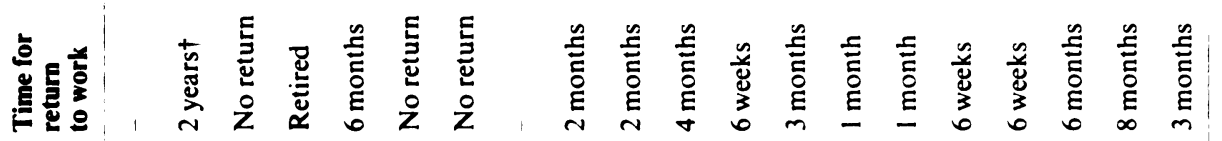

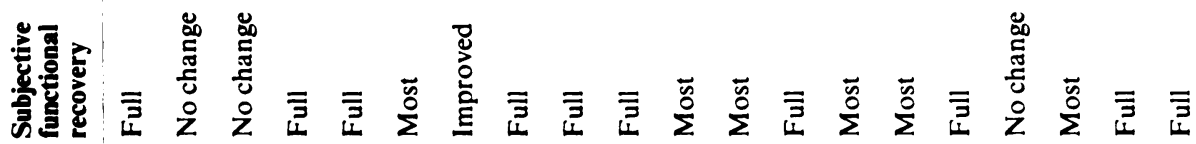

苞

造|

递焉

焉

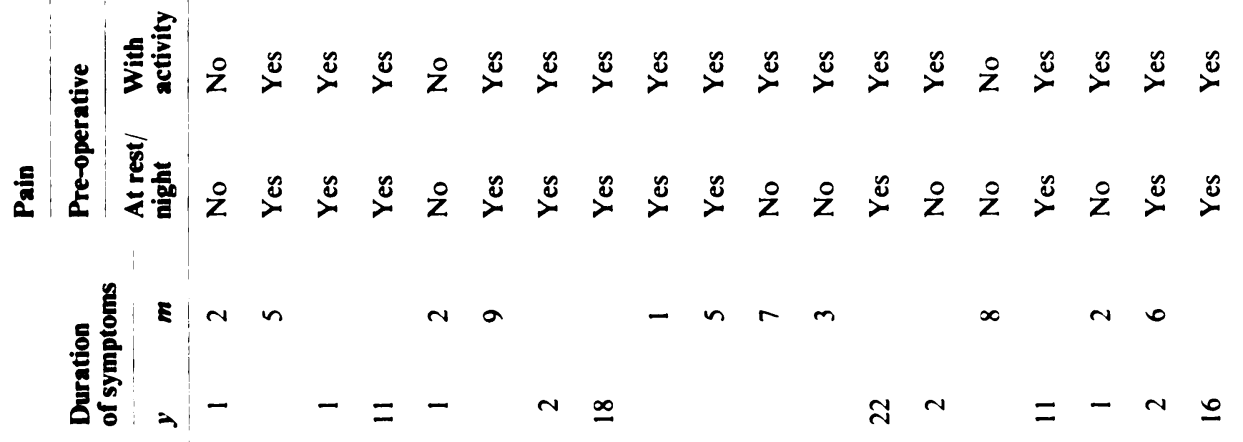

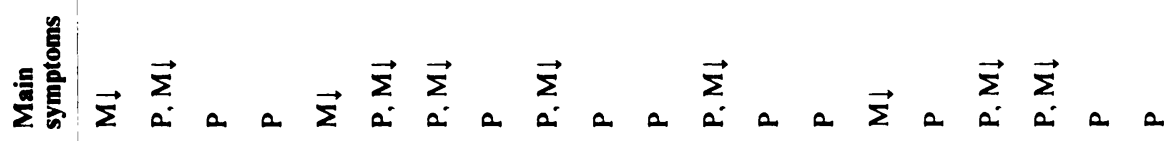

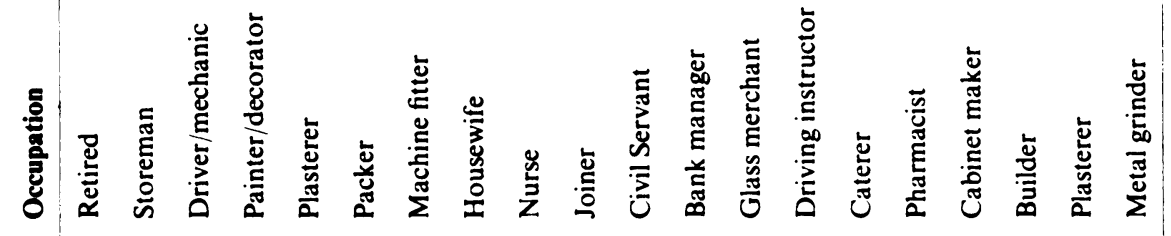

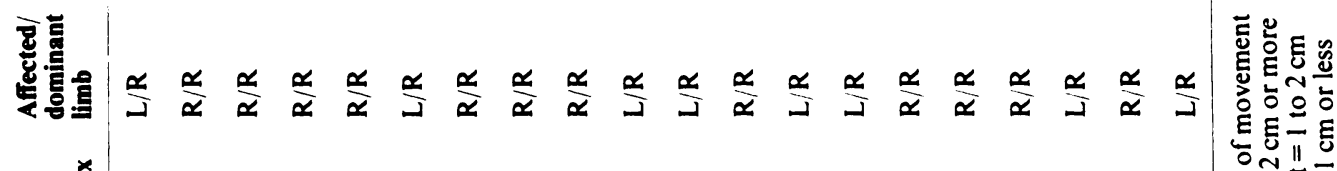

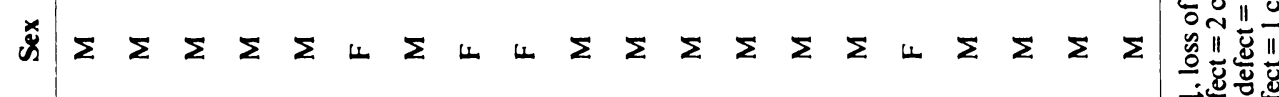

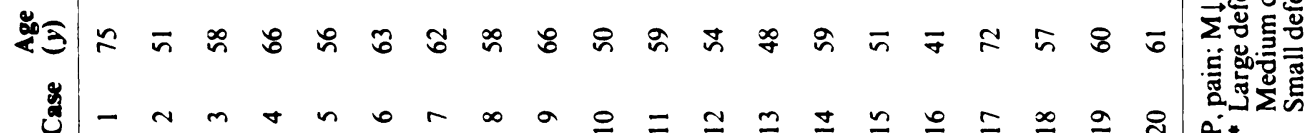




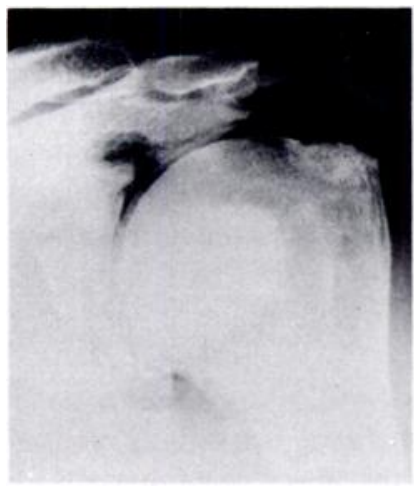

Fig. 1

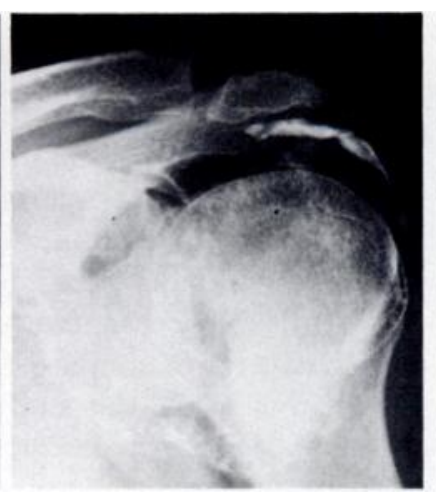

Fig. 2
Case 10. Figure 1-Double-contrast arthrogram performed in 1978 before operation: the torn rotator cuff limited active elevation to only 90 . Figure 2-Three years after operation he could elevate 180 but a defect in the cuff is still clearly visible.

medium in eight and large in two. Figures 1 and 2 show the appearance of the shoulder in Case 10 before operation and three years later. Before operation this patient had only 90 of active elevation; three years after operation he could elevate to 180 , but a defect in the cuff was still clearly visible.

Of the 20 patients, 17 had complete relief of pain; a further two patients had lost their night pain and rest pain, but still had pain on activity, although this was less than before operation. One patient (Case 3) had no relief of pain.

The range of active elevation was full in 15 patients, up to 140 in one patient and 90 or less in four patients (Table I).

Ten patients felt that they had regained full function, six thought they had sufficient function for most activities, one had improved and three were functionally no better. Eighteen patients were working up until the time they entered hospital for operation, 12 returned to their original occupations and were working at the time of review; one returned to work and had subsequently reached retiring age, one had reached retiring age before completion of treatment, one had changed to a lighter job and three did not go back to work (Table I).

The two patients whose rotator cuffs were radiographically intact had complete relief of pain, a full range of active movement and full function. Of the four patients who had 90 or less of active abduction, three had a small and one a large radiographic defect in the rotator cuff. In the remaining 14 patients, all of whom had relief of pain and sufficient function for most or all activities, there were five small, eight medium and one large radiographic defects in the rotator cuff (Table I).

\section{DISCUSSION}

Many tears of the rotator cuff are asymptomatic (Cotton and Rideout 1964), and discussion continues about the value of repairing such tears. Persistent pain is the primary indication for operation (Wolfgang 1978), but there
Table II. Summary of operative findings and procedures

\begin{tabular}{ll}
\hline Case & Findings \\
\hline $1 \quad \begin{array}{l}\text { Large tear in degenerate cuff } \\
\text { Long head of biceps thinned }\end{array}$
\end{tabular}

\section{Procedure}

Direct side-to-side suture Anterior acromioplasty Tenodesis of long head of biceps

2 Large tear anteriorly

Cuff mobilised and resutured to bone

Anterior acromionectomy

3 Large tear at insertion of supraspinatus

Side-to-side suture

Excision of coraco-acromia ligament and outer $1 \mathrm{~cm}$ of clavicle

4 Large avulsion and split of supraspinatus

$5 \quad$ Large tear in supraspinatus

$6 \quad$ Large tear

7 Massive tear at junction of supraspinatus and infraspinatus

8 Large anterior tear

$9 \quad$ Large superoposterior tear

Side-to-side suture

Anterior acromioplasty

Side-to-side suture Anterior acromioplasty

Mobilised and resutured to bone

Infraspinatus and teres minor mobilised and resutured to supraspinatus insertion

Side-to-side suture with carbon fibre supplemen

Anterior acromioplasty

Side-to-side suture with carbon fibre supplement

Anterior acromioplasty

10 Small detachment of supraspinatus with longitudinal tear

$11 \quad$ Large coronal tear extending to supraspinatus insertion

Side-to-side suture and resuture to bone

Side-to-side suture with carbon fibre supplement Anterior acromionectomy

12 Large tear in supraspinatus

13 Small tear at anterior insertion of supraspinatus

14 Large avulsion of infraspinatus and supraspinatus

15 Large anterosuperior tear

Side-to-side suture

Resutured to bone

Excision of coraco-acromial ligament

Side-to-side suture

Anterior acromioplasty

Side-to-side suture with free graft of long head of biceps tendon

16 Small tear. Impingement under the coraco-acromial ligament

Side-to-side suture

Excision of the coraco-acromial ligament

17 Medium anterosuperior tear

$18 \quad$ Large avulsion of supraspinatus

Resutured to bone Anterior acromioplasty

Side-to-side suture and resuture to bone

Anterior acromionectomy

19 Large detachment of supraspinatus

Side-to-side suture and reinsertion to bone

20 Tear in supraspinatus extending to its insertion

Side-to-side suture Anterior acromioplasty

is debate about the relative importance of subacromial decompression as against complete closure of the defect to achieve a good clinical result. Bakalim and Pasila (1975) recommended simple excision of the acromion to 
achieve relief of night pain in selected cases. Apoil et al. (1982) also considered subacromial decompression without repair to be adequate. In a review of a series of rotator cuff repairs from this hospital it was found that when subacromial decompression was included as part of the repair operation, a significantly better relief of pain could be expected (Packer et al. 1983). Other authors (Neviaser 1971; Neviaser, Neviaser and Neviaser 1978; Wolfgang 1978; Neviaser 1980) have stated that it is important to make the repair watertight and have tested this at operation. Post, Silver and Singh (1983) attempted to achieve a watertight seal, but stated that it was more important to restore normal tension in the cuff than to close the defect completely. Several other reports stress the importance of repair without undue tension (Wolfgang 1978; Neviaser 1980; Packer et al. 1983).

In the present series, 18 out of 20 patients who had had operative repair showed an arthrographic leak of contrast medium through the rotator cuff at an average of 30 months after repair. In most of these patients relief of pain was excellent with good restoration of function. In a series of 67 rotator cuff repairs Lundberg (1982) reported 21 arthrographies at follow-up and found leakage through the repaired rotator cuff in seven. He recorded a smaller proportion of good results in these seven than in his total series. He also stated that postoperative arthrography indicates that most completely sutured tendons remain intact; this finding contrasts with ours.

Accurate measurement of the size of a defect in the rotator cuff is not easy with a two-dimensional technique such as radiography and some authors (Neviaser 1980) do not believe that arthrographic measurement is possible. Post, Silver and Singh (1983) found no correlation between the amount of dye leakage and the size of the defect observed at operation; this is not surprising as, with all but the smallest defects, the contrast medium rapidly fills the subacromial bursa. Goldman and Gelman (1978) advocated the use of double-contrast arthrography to demonstrate the size of the tear more effectively; a double-contrast technique has been used exclusively at our institution since 1974 and we would agree with this view. In a recent monograph on shoulder arthrography (Goldman, Dines and Warren 1982) the authors suggest that the width of a tear can usually be evaluated on the film obtained at arthrography; they also state that after a successful repair of the rotator cuff the arthrogram should be essentially normal, but offer no evidence to support this statement. Kilcoyne and Matsen (1983) used arthropneumotomography to demonstrate the size of the cuff tear and found good correlation with the surgical appearances in a grading of small, medium and large tears; they did not define these grades, but the range in width was $0.3 \mathrm{~cm}$ to $4.0 \mathrm{~cm}$. In the present study the occurrence of leakage was recorded as a significant and unequivocal feature. In addition the size of the defect was estimated by the radiologist at fluoroscopy; it was clearly not possible to correlate the estimated size with the operative findings.

In conclusion, this study demonstrates that a good functional result can be achieved after operative repair of the torn rotator cuff, despite the presence of an arthrographic leak. A previous report from this hospital demonstrated that an adequate subacromial decompression was an important part of the repair operation (Packer et al. 1983). It is probable therefore that subacromial decompression and the restoration of a mechanically effective cuff with normal tension are more important than an absolutely watertight repair. Since an arthrographically demonstrable defect persists in many clinically successful repairs, it follows that routine arthrography is unlikely to be helpful in the investigation of the failed repair.

\section{REFERENCES}

Apoil A, Dautry P, Koechlin Ph, Hardy J. The surgical treatment of rotator cuff impingement. In: Bayley I, Kessel L, eds. Shoulder surgery. Berlin etc: Springer-Verlag. 1982:22-6.

Bakalim G, Pasila M. Surgical treatment of rupture of the rotator cuff tendon. Acta Orthop Scand 1975:46:751-7.

Cotton RE, Rideout DF. Tears of the humeral rotator cuff: a radiological and pathological necropsy survey. J Bone Joint Surg $[B r] 1964$ 46-B: 31428 .

Goldman AB, Gelman B. The double-contrast shoulder arthrogram: a review of 158 studies. Radiology 1978;127:655-63.

Goldman AB, Dines DM, Warren RF. Shoulder arthrography: technique, diagnosis and clinical correlation. Boston: Little, Brown \& Co, 1982.

Kilcoyne RF, Matsen FA III. Rotator cuff measurement by arthropneumotography. $A J R$ 1983;140:315-8.

Lundberg BJ. The correlation of clinical evaluation with operative findings and prognosis in rotator cuff rupture. In: Bayley I, Kessel L, eds. Shoulder surgery. Berlin etc: Springer-Verlag, 1982:35-8.

Neviaser JS. Ruptures of the rotator cuff of the shoulder: new concepts in the diagnosis and operative treatment of chronic ruptures. Arch Surg 1971;102:483-5.

Neviaser JS, Neviaser RJ, Neviaser TJ. The repair of chronic massive ruptures of the rotator cuff of the shoulder by use of a freeze-dried rotator cuff. J Bone Joint Surg [Am] 1978;60-A:681-4.

Neviaser RJ. Tears of the rotator cuff. Orthop Clin North Am 1980; 11(2):295-306.

Packer NP, Calvert PT, Bayley JIL, Kessel L. Operative treatment of chronic ruptures of the rotator cuff of the shoulder. J Bone Joint Surg $[B r] 1983 ; 65-B: 171-4$.

Post M, Silver R, Singh M. Rotator cuff tear: diagnosis and treatment. Clin Orthop 1983;173:78-91.

Wolfgang GL. Rupture of the musculotendinous cuff of the shoulder. Clin Orthop 1978; 134:230-43. 